\title{
Los inicios de la Psicotecnia en España
}

RESUMEN: El presente trabajo tiene por objetivo determinar los orígenes de la Psicología Aplicada o Psicotécnia en España. Se describen las instituciones más relevantes de esta primera época en cada una de las dos ciudades donde esta labor adquirió su mayor preponderancia: Madrid y Barcelona. Se da cuenta de las figuras que llevaron a cabo este nacimiento psicológico, haciendo referencia a sus campos de aplicación: psicoeducativo u orientación profesional.

PALABRAS CLAVE: Psicología Aplicada. Historia de la psicología en España. Orientación profesional

\section{Introducción}

El objetivo general de la Psicología aplicada o Psicotecnia es transferir las investigaciones psicológicas a la vida práctica a través de una aplicación concreta. En su nacimiento va a estar ligada, en mayor medida, a la orientación profesional y la pedagogía y, como afirma R. Zazzo (1), lo esencial es subrayar que el terreno industrial y el educacional fueron históricamente los dos primeros y principales ámbitos de la psicología aplicada.

El nombre de Psicotecnia se debe al psicólogo personalista alemán William Stern (1871-1938), que lo empleó por primera vez en 1903, fundando años más tarde (1907) la primera revista de psicología aplicada en colaboración con Otto Lippmann. En esta época hay que destacar en Francia los trabajos sobre selección de personal de J.M. Lahy, que fue además creador de varios aparatos psicotécnicos.
SUMMARY: The objetive of this work is to accomplish the origin of applied Psychology in Spain. The most outstanding institutions of this first period are described in each one of the two cities where this labour has acquired his greatest preponderance: Madrid and Barcelona. It's analyzed the figures who carried out this psychological birth, particulary their fields of application: psychoeducative or professional orientation.

KEY WORDS: Applied Psychology. History of the Spanisch Psychology. Professional orientation.

En 1908, en Bélgica, Christiaens fundó con Decroly el primer gabinete de orientación profesional de Europa. Paralelamente, en el mismo año, Parsons crea en Boston un Servicio de Consejo y Orientación para los muchachos que terminaban el período escolar.

Algunos años más tarde, en 1918, Edouard Claparede creó, junto a Pierre Bovet, el Gabinete de Orientación Profesional del Instituto Jean Jacques Rousseau, en Ginebra, prototipo de los futuros laboratorios de investigaciones psicotécnicas (2).

Por lo que respecta a España, resulta difícil establecer con exactitud los comienzos de la Psicología aplicada o Psicotecnia. Pero no cabe duda de que a partir de 1910, de modo progresivo y sigiloso, se va formando un creciente y paulatino interés por la intervención técnica 
en el campo de la psicología. En estos comienzos dos ámbitos de actuación destacan sobremanera como ocurre en el resto de Europa: la Psicopedagogía y la Orientación Profesional.

Los historiadores de la psicología han centrado, casi exclusivamente, sus investigaciones en la producción, gestación y desarrollo de las ideas y concepciones teóricas de los distintos autores o escuelas, olvidando frecuentemente el marco institucional concreto donde se aplican así como sus resultados concretos y operativos. Los historiadores se han venido ocupando del desarrollo y la sucesión lógica de las ideas olvidando, por lo general, ese contexto en el que se desarrollan y existen (3).

Se analiza a continuación las primeras instituciones que destacaron en los inicios de la psicología aplicada en España. Instituciones que fueron pioneras en la utilización de métodos e instrumentos psicológicos, y que ejercieron su influencia en otras instituciones del país.

Dos ciudades van a copar en exclusividad la organización de este tipo de instituciones: Barcelona y Madrid.

\section{La Psicotecnia en Barcelona.}

Son tres las instituciones psicológicas que en el primer tercio de siglo destacan en Barcelona:

a) El Secretariado de Aprendizaje.

Fue el primer Servicio de Orientación Profesional que empezó a funcionar en España allá por el año 1915. Su finalidad consistía en orientar a los trabajadores jóvenes en la elección de una profesión, atendiendo a las aptitudes y preferencias de cada sujeto. Para ello se reque- ría conocer los datos exactos de cada uno de los oficios; observar las aptitudes físicas, intelectuales y morales de los chicos y ejercer función de patronato, es decir, vigilar el desarrollo físico e intelectual del aprendiz, así como su perfeccionamiento profesional.

El Secretariado se dividía en tres secciones: Sección de Información, Sección Antropométrica y Sección Psicométrica. Funcionaba en la práctica como un consultorio de orientación tras la realización de un examen medico-fisiológico y otro psicológico, ya que el progreso de la fisiología y la psicología del trabajo planteó la necesidad de dar una base científica a las consultas vocacionales que allí se realizaban (4).

b) El Instituto de Orientación Profesional.

En 1918, el Secretariado de Aprendizaje se vio en la necesidad de ampliar sus actividades y se transformó en un Instituto de Orientación Profesional dependiente de la Mancomunidad de Cataluña y del Ayuntamiento de Barcelona. Su estructura queda dividida en las secciones de Información, Médico-Antropométrica, Psicométrica y Estadística.

Se dota al Instituto de mayor personal técnico y de esta manera, en 1919 , son contratados los doctores Luis Trias de Bes (1895-1974) y Emilio Mira y López (1896-1964) como responsables de las secciones de Antropometría y Psicometría respectivamente. Mira y López, nacido en Cuba y formado en Barcelona es sin duda una de las figuras más importantes de la psicología española de la época. Médico, psiquiatra y posiblemente el primer psicólogo que ha tenido nuestro país (5). Muy pronto logró establecer relaciones con los 
HISTORIA

mejores investigadores europeos de su campo, abriendo la psicología española a las principales innovaciones técnicas de la época. De esta manera impartieron seminarios en Barcelona figuras internacionales tan relevantes como Kohler (18871967), Michotte (1881-1965), Pieron (1881-1964), etc.

Como señala Ruben Ardila (6), probablemente Mira y López fue el primer hispanoparlante en interesarse en la obra del fundador del conductismo J.B. Watson, publicando en 1921 un artículo sobre esta corriente psicológica. De la misma manera fue uno de los primeros en España en estudiar a Freud. En 1925 trabajó con el test de Rorschach y con la tipología de Kretschmer, que constituían grandes novedades en la psicología de su época.

Una de sus contribuciones técnicas más importantes lo constituye su Psicodiagnóstico Miokinético (PMK), expuesto en 1940 que tiene por finalidad la exploración de las tendencias conativas de la personalidad.

El Instituto alcanzó tal proyección que, en 1922 y 1930, se celebraron en Barcelona la II y IV Conferencia Internacional de Psicotecnia, que tuvieron como presidente a Claparede (1873-1940) y a la que asistieron los principales psicólogos europeos.

c) El Laboratorio Psicológico-pedagógico del Colegio Máximo de San Ignacio de Sarriá. Este laboratorio fue creado en 1925 por el jesuita Fernando $M^{a}$ Palmés con la finalidad de orientar y centralizar las observaciones de los Gabinetes Paidométricos, que fueron organizándose en los colegios de segunda enseñanza de la Compañía de Jesús. Los colegios de la Compañía tenían la costumbre desde antiguo de realizar informes acerca de sus alumnos donde se reflejaban sus principales características escolares y personales. La tarea del P. Palmés consistió en organizar la obtención de dichos informes de una manera más científica, atendiendo no sólo al rendimiento escolar del alumno, sino también a todos los aspectos fisiológicos y psicológicos de los muchachos (7).

El estudio realizado a los alumnos se utilizaba para establecer más adecuadamente su desarrollo educativo por una parte y por otra para la orientación profesional del alumno al terminar su aprendizaje en el colegio.

Para realizar el estudio psicológico se exploraba la inteligencia y la personalidad. La primera se medía con un instrumento validado como era el Test de BinetSimon. Sin embargo el estudio de la personalidad se realizaba, de una manera menos rigurosa, con un método ideado por el jesuita holandés J. van Ginneken mediante el que se obtenía un perfil psicológico del alumno que se explicitaba por medio de una psicografía circular (8).

d) El Laboratorio de Experimentación Psicológica de la Junta de Protección a la Infancia. A raíz de la promulgación de la Ley de Protección a la Infancia de 1904 comenzó a funcionar en Barcelona, en 1914 , un albergue para niños abandonados, dependiente de la Junta de Protección a la Infancia. Posteriormente, en 1917, se le dotó de un departamento de observación que incluía el Laboratorio de Experimentación. Estaba dirigido por Luis $\mathrm{M}^{\mathrm{a}}$ Folch y Torres, quién inspirándose en los procedimientos de M. Rouvroy, director del Departamento Central de Observación de Moll en Bélgica, comenzó a estudiar, se 
gún sus métodos, la psicología de los menores tutelados. Por este trabajo puede con razón ser considerado como el precursor de la orientación psicológica de la Protección de Menores de Barcelona, y aun de España (9).

Conviene señalar, en este apartado, que en 1916 Gabriel $\mathrm{M}^{a}$ de Ybarra, en una visita realizada a Barcelona, conoció el sistema empleado en este Laboratorio e influyó para que la legislación de menores, que en aquel entonces se estaba elaborando, recogiese la exploración psicológica de los menores. Aquí tuvo referencias de $\mathrm{M}$. Rouvroy que más adelante conocerían los Padres Terciarios Capuchinos que se dedicaron a la psicología, visitando el propio Centro de Moll.

A Folch lo encontramos años más tarde participando activamente en la II Conferencia de Psicotecnia, celebrada en Barcelona en 1922 y a la que ya hemos hecho referencia.

\section{La Psicotecnia en Madrid.}

La psicología aplicada española está indisolublemente unida a las figuras de Mira, Rodríguez Lafora, comentados anteriormente, así como a José Germain (1898-1986). Germain es la figura puente entre los pioneros de la psicología espanola y los maestros de la actual generación de psicólogos. Discípulo de Rodríguez Lafora, conectó con buena parte de los psicólogos europeos de la época al realizar estudios con Claparede, Kohler, Rupp, Janet, Toulouse, etc. y reorganizó la psicología en España tras la Guerra Civil. Entre sus discípulos se encuentran quienes han dirigido la psicología en España en las últimas décadas: Mariano Yela,
José Luis Pinillos, Miguel Siguan, Francisco Secadas y Jesusa Pertejo, entre otros. José Germain (10) trabajó asiduamente en la consolidación de la psicotecnia y orientación profesional. En 1930 es nombrado director del Instituto Psicotécnico de Madrid. Acabada la Guerra Civil, fundó en 1946 la más importante revista científica española, la Revista de Psicología General y Aplicada. En 1952 funda la Sociedad Española de Psicología y dos años más tarde la Escuela de Psicología para posgraduados, que sería el germen de las futuras facultades de Psicología en España (11).

Se desarrollarán a continuación algunas de las instituciones donde se cuajó la psicología aplicada en Madrid.

a) El Patronato Nacional de Anormales. Su creación en 1914 desencadenó un amplio movimiento en pro de la educación especial. Su primer secretario fue Nicolás Achucarro (1880-1918), discípulo de Cajal y amigo y colaborador de Lafora. Creó una consulta pública para deficientes y organizó cursos para maestros sobre psicología y pedagogía de la subnormalidad. Se hizo de este modo tema candente el diagnóstico psicopatológico de la subnormalidad (12). En esta línea se encontraba la Inspección Médico-escolar (1913) con Saínz de los Terreros y César Juarros o el Instituto Médico-Pedagógico de Lafora en Carabanchel.

b) El Servicio de Orientación Profesional. Fue el primero de este tipo que se creó en Madrid en 1922 y dependía del Instituto de Reeducación de Inválidos del Trabajo. Constituyó la célula inicial, a partir de la cual, en sucesivas etapas, se fue organizando primero el Instituto de Orientación y Selección Profesional, luego el 
HISTORIA

Instituto Psicotécnico y por último el Instituto Nacional Psicología Aplicada y Psicotecnia (13).

Esta Sección de Orientación tenía como función seleccionar a los inválidos más susceptibles de reeducación y a distribuirlos por los diversos talleres que estaba instalando el Instituto para la Reeducación Profesional y la Readaptación al Trabajo Industrial. Allí se experimentaron problemas de psicomotricidad y ergología, que sirvieron para los primeros cursos organizados en Madrid para la formación complementaria de médicos del trabajo (14).

Estaba dirigido por César de Madariaga y en él trabajaban Mercedes Rodrigo como psicóloga y José Mallart como psicotécnico. Estos dos últimos provenían del Instituto Juan Jacobo Rousseau, donde se habían formado bajo la dirección de Claparede (1873-1940). Mercedes Rodrigo por su parte fue la primera psicóloga española en trabajar con Piaget (1896-1980).

Posteriormente comenzó a colaborar con este Servicio José Germain, al hacerse cargo de la consulta de neurología del Instituto de Inválidos. Este equipo, además de las tareas propias de orientación, realizó una enorme labor de adaptación y creación de test e instrumentos psicológicos: Fueron muchos los test que entonces surgieron desde el perceptotakímetro de Madariaga, la prueba de atención distribuida con ayuda de un kimógrafo y los diversos test de aptitudes mecánicas que por entonces manejaban los psicotécnicos y que Mallart supo adaptar o idear con ingenio; así como test de inteligencia, conocimientos pedagógicos y de personalidad que desarrollaron J. Germain y Mercedes Rodrigo. c) Instituto de Orientación y Selección Profesional. En 1924, el Instituto de Reeducación de Inválidos y el Instituto de Orientación Profesional de Barcelona pasaron a denominarse Instituto de Orientación y Selección Profesional, dependientes ambos del Ministerio de Trabajo. Recibieron atribuciones de carácter general y poder llevar a cabo, ambos, labores de investigación y control técnico de los servicios oficiales de orientación que empezaban a crearse en las principales provincias. Su finalidad era la mejora de los rendimientos en el trabajo y la efectividad profesional. A estos institutos acudían los jóvenes en busca de consejo para comenzar estudios o realizar ciertos aprendizajes (15).

Esta expansión a nivel nacional de la psicología aplicada fue posible al ser nombrado Cesar de Madariaga Director General de Comercio en el Ministerio de Trabajo, que encargó a Germain y Mira el estudio de la organización de todo el sistema de orientación profesional en España.

En este período, en el Instituto de Madrid, Mallart trabajaba en su función de psicotécnico, en temas de orientación en el ambiente industrial y social. Por su parte Mercedes Rodrigo y José Germain se dedicaban más a preparar material de exploración y muy especialmente tests de inteligencia. De esta colaboración salió, entre otros, la adaptación del test de Ballard que fue ensayado por los dos en casi todas las escuelas municipales de Madrid (1925) y cuyos resultados con la clasificación y estudios correspondientes fueron destruidos durante la Guerra Civil (16). Mejor suerte corrió la adaptación de las pruebas de inteligencia de Terman que realizaron 
HISTORIA

posteriormente. En este campo de investigación estudiaron también tests de atención, de memoria, de aptitud mecánica y se protocolizaron diversos tests manuales.

Este equipo del Instituto estuvo en constante relación con la psicología europea del momento, como lo demuestran su participación en las conferencias internacionales de psicotecnia de París, Utrecht, Barcelona, Londres, etc., donde establecen contacto científico con Claparede, Toulouse, Michotte, y otros. Así mismo, como complemento de la formación profesional, se organizan en el Instituto ciclos de conferencias y seminarios donde aparecen los nombres de Pierón (París), Rupp (Berlín), Piaget (Ginebra) y Giese (Stuttgart), por ejemplo (17).

Esta actividad internacional del Instituto hace que se convierta en un centro intelectual y científico de sumo interés, por lo que era asiduamente visitado por figuras relevantes de la época como Sacristán, Gayarre y Ortega y Gasset.

El punto de vista epistemológico de los miembros del Instituto respecto a la psicología aplicada, en general, y a la orientación profesional, en particular, queda reflejado clarificadoramente en las siguientes palabras de J. Germain (18): Creo que la Orientación Profesional produce todos sus efectos beneficiosos para el individuo y para la sociedad cuando al estudio del nivel de inteligencia y al conocimiento de las aptitudes y los intereses, se une un estudio de la personalidad, de sus rasgos característicos y consecuentemente de su capacidad de adaptación, de encaje en las diversas profesiones y oficios. Ese es el momento de establecer el contacto con el mundo de los estudiantes, del trabajo, el mundo del empleo y el mundo social. d) El Instituto Psicotécnico. En Julio de 1930, una real orden cambió la denominación de los Institutos de Orientación Profesional por la de Institutos Psicotécnicos, que pasan a depender, un años más tarde, del Ministerio de Instrucción Pública. De esta manera se ampliaron oficialmente los horizontes de la psicología aplicada en España, hasta entonces por completo reducidos al sector laboral. Y a partir de este momento se extendió su campo de acción hacia todos los sectores y campos de la enseñanza (4).

Esta amplitud de miras y campos de actuación se aprecia en la propia definición que de Psicotecnia aceptaban los miembros del Instituto: Se entenderá por Psicotecnia la aplicación de los métodos y resultados de la Psicología a la consecución de fines prácticos en todas las esferas de la vida humana (19).

El Instituto Psicotécnico de Madrid se transformó posteriormente en Instituto Nacional de Psicotecnia por decreto de marzo de 1934, siendo el órgano rector de los servicios nacionales de psicología aplicada y psicotecnia. Prueba de la pujanza científica de esta institución es el hecho de que la Unión Internacional de Psicología Científica la designara para organizar el XII Congreso Internacional de 1936 en Madrid. Estaba previsto que la presidencia de honor la ocupase Cajal y Ortega y Gasset, siendo la presidencia efectiva para Mira y la secretaria para Germain. Los avatares de la Guerra Civil hicieron que finalmente se celebrase en París.

Al finalizar la Guerra Civil y producirse el exilio de los principales representantes de la psicología en España anteriormente aludidos, el Instituto fue dirigido por Ibarrola hasta el año 1950, en que 
asumió de nuevo la dirección Germain. En 1940 comenzó a publicarse la revista de Psicotecnia que daría paso en 1946 a la Revista de Psicología General y Aplicada donde junto a los principales autores internacionales comienzan a aparecer las publicaciones de las figuras que marcarían la psicología actual y conseguirían su institucionalización definitiva.

En 1952 se crea la Sociedad Española de Psicología y en 1953 la Escuela de Psicología y Psicotecnia en la Universidad de Madrid, terminando este proceso con la aprobación de la licenciatura de psicología (1968) y el establecimiento de facultades universitarias independientes en 1980

\section{Discusión.}

A partir de la Restauración monárquica en 1875, en la persona de Alfonso XII (1857-1885), se inicia un período de mayor estabilidad política en España e inaugura (con sus problemas, tensiones, etc.) la plataforma contemporánea más próxima a nuestra inmediata base histórica (20). Se va perfilando, por otra parte, una etapa más tolerante, civilista y plural aunque no exenta de fuertes confrontaciones ideológicas. La monarquía de la Restauración fue la estructura política más estable erigida por el liberalismo español del siglo XIX, aunque tal estabilidad se basaba en un valor en baja: el ansia de vivir, ese deseo de lograr una vida apacible tras la anarquía (21).

En este período van a persistir dos ideologías radicalmente contrapuestas en la manera de concebir el mundo y por lo tanto en sus presupuestos sociales y culturales: por un lado el institucionalismo krausopositivista y por otro el neoescolasticismo. Ambas posturas en pugna dialéctica van a propiciar, aunque en distintos grados, la conexión con corrientes científicas e intelectuales vigentes en Europa. Como consecuencia, la ciencia psicológica española logra contactar de modo efectivo con el nivel científico de otros países, logrando incluso ciertos desarrollos originales.

Desgraciadamente, la Guerra Civil española (1936-1939) puso fin a este movimiento, y tuvo dramáticas consecuencias para la nueva Ciencia de la Psicología. Todas las revistas dejaron de publicarse, y la mayor parte de los intelectuales y científicos tuvieron que marchar al extranjero, a Europa o Latinoamérica. La arena española fue ocupada por una psicología escolástica, vieja, caduca y autárquica, sin ningún lazo de conexión con la psicología aplicada que se hacía en Occidente (37). 


\section{BIBLIOGRAFIA}

(1) Zazzo R., Tratado de Psicología del Niño. Historia y Generalidades, Madrid, Morata, 1978.

(2) Baumgartem-Tramer F., «Informe sobre la Asociación Internacional de Psicotecnia: Discurso del secretario», Revista de Psicología General y Aplicada, 1951, 6, 19 30.

(3) Peiró JM. y Carpintero H., «Los primeros laboratorios de psicología y su influencia en la aparición de esta ciencia», Análisis y Modificación de Conducta, 1978, 4-5, 129 . 158.

(4) Cerdá E., Psicología aplicada, Barcelona, Herder, 1960.

(5) Carpintero H., «La Psicología en España: una síntesis», en Mayor J. y Pinillos JL. (eds), Tratado de Psicología General. Historia, Teoría y Método, Madrid, Alhambra, 1989: 329-351.

(6) Ardila R., Los pioneros de la psicología, Buenos Aires, Paidós, 1971.

(7) Kirchner M., «Historia de la psicología aplicada en Barcelona (1916-1936)», Anuario de Psicología, 1979, 20, 3-22.

(8) Palmés FM., «Funcionamiento del laboratorio médico-psicológico del colegio Máximo de San Ignacio en Barcelona", Anals d'Orientació Professional, 1930, 4, 275-285.

(9) Palmés FM., «Prólogo», en Piquer y Jover JJ., El niño abandonado y delincuente, Madrid, Consejo Superior de Investigaciones Científicas, 1945, 1-11.

(10) Germain J., «Para la pequeña historia de la Psicología en España», Revista de Psicologia General y Aplicada, 1954, 32, 633642.
(11) Mora JA., El Dr. José Germain: hitos principales de su biografía (1897-1986), Málaga, Edinford, 1992.

(12) Carpintero H., «La evolución del psicodiagnóstico en España», Evaluación Psicológica, 1989, 5(1), 3-21.

(13) Germain J., «Reorganización del Instituto Nacional de Psicotécnia y de sus delegaciones», Revista de Psicología General y Aplicada, 1955, 35, 649-652.

(14) Mallart J., Psicología industrial y organizacional, Madrid, Artes Gráficas y Ediciones, 1981.

(15) Fernández Zúñiga A. y García Tuñón R., «Apuntes para una historia de la psicología en España», Cuadernos de Psicología, 1976, 3(4), 26-30.

(16) Germain J., «Autobiografía I», Revista de Historia de la Psicología, 1980, 1(1), 7 32.

(17) Mallart J., «Memorias de un aspirante a psicólogo", Revista de Historia de la Psicología, 1981, 2(2), 91-123.

(18) Germain J., «Autobiografía II», Revista de Historia de la Psicología, 1980, 1(2), 139170.

(19) Germain J., «La formación del personal técnico para los servicios y laboratorios de Psicología y Psicotecnia», Revista de Psicotecnia, 1942, III(12), 527-553.

(20) Jutglar A., Ideologías y clases en la España contemporánea (1874-1931) II, Madrid, Cuadernos para el Diálogo, 1969.

(21) Carr R., España 1908-1936, Barcelona, Ariel, 1969.

(22) Peiró JM y Carpintero H., «Historia de la Psicología en España a través de sus revistas especializadas», Revista de Historia de la Psicología, 1981, 2(2), 143-181.

Autores:

Vicente Sánchez Vázquez. Psicólogo Clínico

Teresa Guijarro Granados. Psiquiatra

Unidad de Salud Mental Infanto-Juvenil. Hospital «Reina Sofía». Córdoba

Servicio Andaluz de Salud

Fecha de recepción: 4-X-1999 